\title{
Osteogenic effect of bone marrow mesenchymal stem cell-derived exosomes on steroid-induced osteonecrosis of the femoral head
}

This article was published in the following Dove Press journal:

Drug Design, Development and Therapy

\author{
Shanhong Fangl,* \\ Yongfeng $\mathrm{Li}^{2, *}$ \\ Peng Chen' \\ 'Department of Orthopedic Surgery, \\ The First Affiliated Hospital of Fujian \\ Medical University, Fuzhou 350005, \\ China; ${ }^{2}$ Department of Bone Surgery, \\ Fujian Medical University, Fuzhou \\ 350005, China \\ *These authors contributed equally \\ to this work
}

Background: Animal studies have demonstrated the therapeutic effect of mesenchymal stem cells (MSCs) on osteogenesis, but little is known about the functions of exosomes (Exos) released by bone MSCs (BMSCs). Here, we investigated the effect of BMSC Exos on steroid-induced femoral head necrosis (SFHN) and explored the vital genes involved in this process.

Materials and methods: BMSCs were isolated from healthy and SFHN rats. BMSC Exos were isolated using the Exosome Precipitation Kit and characterized by transmission electron microscopy and Western blotting. SFHN BMSCs were incubated with Exos from healthy BMSCs. Osteogenic ability was assessed by oil red O staining and alizarine red staining. Differentially expressed genes (DEGs) induced by Exos were screened using the Osteogenesis RT ${ }^{2}$ Profiler PCR Array. The effect of upregulated Sox 9 was examined using lentivirus-mediated siRNA.

Results: The results revealed that BMSC Exos were 100-150 nm in size and expressed CD63. Moreover, BMSC Exo-treated SFHN cells exhibited suppressed adipogenesis compared to model cells. PCR array showed that eleven and nine genes were upregulated and downregulated, respectively, in the BMSC Exo-treated SFHN cells compared to the model group. Among the DEGs, osteogenesis-related genes, including Bmp2, Bmp6, Bmpr1b, Mmp9, and Sox9, may play important roles in SFHN. Furthermore, the DEGs were mainly involved in immune response, osteoblast differentiation, and in the transforming growth factor- $\beta /$ bone morphogenetic protein signaling pathway. The level of the SOX9 protein was upregulated by Exos, and Sox 9 silencing significantly decreased the osteogenic effect of BMSC Exos.

Conclusion: Our data suggest that Exos derived from BMSCs mainly affect SFHN osteogenesis, and this finding can be further investigated to develop a novel therapeutic agent for SFHN.

Keywords: exosome, bone marrow mesenchymal stem cells, steroid-induced femoral head necrosis, gene expression, ostosis

\section{Introduction}

Steroid-induced femoral head necrosis (SFHN) is a major metabolic disorder that occurs following long-term hormone drug use, which decreases the vascular supply to the femoral head and osteocytes and causes bone marrow component death, resulting in internal structural changes, collapse of the femoral head, and hip joint dysfunction. Approximately $75 \%$ of patients suffer from femoral head collapse within 3 years of SFHN diagnosis, and more than $65 \%$ of patients must undergo total hip arthroplasty. While many recent studies have examined the pathogenesis of SFHN, the underlying molecular mechanisms remain unclear.

Bone marrow mesenchymal stem cells (BMSCs) are pluripotent cells that can differentiate into various cells types, such as chondrocytes, osteoblasts, and adipocytes. ${ }^{1-3}$
Correspondence: Peng Chen

Department of Orthopedic Surgery, The First Affiliated Hospital of Fujian Medical University, No 20, Chazhong Road, Taijiang District, Fuzhou 350005, China Email 290633950@qq.com
Drug Design, Development and Therapy 2019:13 45-55

45 
Numerous diseases, including osteoporosis and osteonecrosis, are significantly associated with disbalances between osteogenic and adipogenic differentiation of BMSCs. ${ }^{4,5}$ Moreover, treatment of patients with a high dose of corticosteroids can lead to osteonecrosis of the femoral head by enhancing ischemia and intraosseous pressure in the femoral head. ${ }^{6}$ Additionally, BMSCs treated with steroids accumulated tyiclyceride, indicating that steroids increased the adipogenic differentiation of BMSCs. ${ }^{7}$ A recent study showed that osteonecrosis of the femoral head is mainly caused by an increased differentiation of BMSCs into adipocytes and twisted bone formation. ${ }^{8}$

Exosomes (Exos) are 30-150 nm diameter membrane vesicles that originate from multivesicular bodies. ${ }^{8,9}$ Exos have been isolated from various cells and body fluids, such as endothelial cells, immune cells, blood platelets, smooth muscle cell, blood, saliva, urine, and cerebrospinal fluid, ${ }^{10}$ and they contain cellular plasma, lipids, proteins, and nucleic acids. ${ }^{11-14}$ Exos in peripheral blood mononuclear cells are involved in immune surveillance. ${ }^{7}$ Exos derived from dendritic cells may activate the immune system and have been used in the therapy of tumors. ${ }^{8,9}$ Mesenchymal stem cell (MSC)-derived Exos exhibited anticarcinogenic action by suppressing mitogen-activated protein kinase signaling pathways. ${ }^{11}$ Moreover, natural killer cell-derived Exos showed toxicity toward melanoma cells. ${ }^{12-14}$ Although BMSCs have active self-proliferative and multidifferentiated capacity, little is known about the function of Exos derived from BMSCs.

In this study, we examined the therapeutic effect of BMSC Exos on SFHN by establishing an SFHN cell model and treatment with BMSC Exos. Moreover, we used the Osteogenesis $\mathrm{RT}^{2}$ Profiler PCR Array to analyze the mechanism of the effects of BMSC Exos.

\section{Materials and methods Isolation of BMSC Exos}

BMSCs were isolated from healthy and SFHN rats as a previously study. ${ }^{15}$ Exos from the cell supernatants were isolated as previously described ${ }^{16}$ using the ExoQuick ${ }^{\text {TM }}$ Plasma Prep and Exosome Precipitation Kit (Cat\# EXOQ5TM-1, SBI System Biosciences, Mountain View, CA, USA). Briefly, the BMSCs were cultured in T75 culture bottles until 80\% confluency was reached, after which the culture medium was changed to $100 \mathrm{~mL}$ serum-free medium, and the supernatant was collected after 48 hours of culture. The supernatant was mixed with $63 \mu \mathrm{L}$ of ExoQuick Exosome Precipitation Solution after centrifugation at $3,000 \times g$ for 15 minutes. The ExoQuick mixture was centrifuged at $1,500 \times g$ for 30 minutes at $20^{\circ} \mathrm{C}$. The residual ExoQuick solution was centrifuged at $1,500 \times g$ for 5 minutes to pellet the Exos. Finally, the exosome pellet was resuspended in $100 \mu \mathrm{L} 1 \times$ PBS.

\section{Transmission electron microscopy (TEM)}

The Exos were fixed in 2\% paraformaldehyde solution, processed into ultrathin sections, and labeled with an anti-CD63 antibody (ab108950, Abcam, Cambridge, UK). All sections were examined by transmission electron microscopy, and images were recorded with an AMT 2 k CCD camera.

\section{Western blotting}

Exos from the cell supernatant were prepared by lysis in RIPA lysis buffer with proteinase inhibitor (Promega, Madison, WI, USA). The total protein level in each sample was measured using a BCA assay kit (Pierce Biotechnology, Inc., Rockford, IL, USA). The same quantity of proteins from each sample were separated by $10 \%$ SDS-PAGE and transferred to polyvinylidene fluoride membranes (Millipore, Billerica, MA, USA). The membranes were blocked with 5\% nonfat milk solution in Tris-buffered saline containing 0.05\% Tween 20 (TBST) (0.1\% Tween 20), and then incubated with antibodies for mouse anti-CD63 (1:1,000; ab108950, Abcam), mouse anti-Sox9 (1:1,000; ab185230, Abcam), and glyceraldehyde3-phosphate dehydrogenase (GAPDH) (1:1,000; ab9482, Abcam) for 12 hours at $4^{\circ} \mathrm{C}$. The membranes were washed and incubated with horseradish peroxidase-conjugated goat-antimouse IgG (Santa Cruz Biotechnology, Inc., Dallas, TX, USA) for 2 hours. Protein bands were detected using enhanced chemiluminescence reagent (Thermo Fisher Scientific, Waltham, MA, USA) and visualized via a ChemiDoc MP system (Bio-Rad, Hercules, CA, USA).

\section{Exosomal treatment and steroid induction}

Rat BMSCs were cultured in DMEM/F12 containing 10\% FBS and $1 \%$ penicillin/streptomycin (Procell, Wuhan, China) in a 24-well plate at a density of $5 \times 10^{4}$ cells/well in a humidified incubator containing $5 \% \mathrm{CO}_{2}$ at $37^{\circ} \mathrm{C}$. The cells were divided into three groups in triplicate: SFHN model group (SFHN BMSCs induced with $10^{-7} \mathrm{M}$ dexamethasone), BMSC Exos group (SFHN BMSCs treated with healthy BMSC Exos, and induced with $10^{-7} \mathrm{M}$ dexamethasone), and control group (healthy BMSCs induced with $10^{-7} \mathrm{M}$ dexamethasone). For all groups, after incubation in basic medium for 24 hours, the medium was changed to DMEM/F12 containing $10 \% \mathrm{FBS}, 1 \%$ penicillin/streptomycin, and $10^{-7} \mathrm{M}$ dexamethasone, and the medium was replaced every 3 days. After induction for 18 days, the medium of the Exos group was replaced with medium not containing dexamethasone, and then $5 \mu \mathrm{g}$ BMSC Exos was 
added. While the control and model group were induced with medium containing dexamethasone. All groups were cultured for 3 days for oil red $\mathrm{O}$ staining and alizarine red staining.

\section{Oil red $O$ staining}

Cells were washed with PBS and fixed with 4\% paraformaldehyde for 30 minutes at $20^{\circ} \mathrm{C}$, and then stained with oil red O solution (Jiancheng Biotechnology, Nanjing, China) for 1 hour at $20^{\circ} \mathrm{C}$. The stained cells were washed with PBS and observed using a microscope.

\section{Alizarine red staining}

Cells were washed three times with PBS and stained with $0.1 \%$ alizarin red $\mathrm{S}$ in distilled water $(\mathrm{pH} 4.2)$ for 1 hour at $20^{\circ} \mathrm{C}$. After staining, the cells were washed three times with distilled water and observed using a microscope. For quantification of stained cells, integrated OD of lipid droplets and osteogenesis was counted using Image-pro plus.

\section{Osteogenesis $\mathrm{RT}^{2}$ Profiler PCR Array}

To explore the genes involved in the effects of BMSC Exos on SFHN, an osteogenesis PCR array was performed in the model and BMSC Exos group in triplicate. Total RNA was isolated utilizing TRIzol reagent (Invitrogen, Carlsbad, CA, USA) according to the manufacturer's instructions. The PCR array was performed by Yingbio Technology Co., Ltd. (Shanghai, China). Briefly, RNA integrity was tested via standard denaturing agarose gel electrophoresis. Total RNA $(1 \mu \mathrm{g})$ was reverse-transcribed utilizing the $\mathrm{RT}^{2}$ First Strand Kit (330401, Qiagen, Hilden, Germany), and cDNA was diluted with $\mathrm{ddH}_{2} \mathrm{O}$ to a volume of $100 \mu \mathrm{L}$, and then $1 \mu \mathrm{L}$ cDNA was used for each reaction in the Rat Osteogenesis RT $^{2}$ Profiler PCR Array (PARN-026Z, Qiagen). Real-time PCR (RT-PCR) was performed using $\mathrm{RT}^{2}$ SYBR Green Mastermix (Qiagen, 330401) under the following PCR conditions: $95^{\circ} \mathrm{C}$ for 10 minutes, followed by 40 cycles of $95^{\circ} \mathrm{C}$ for 15 seconds, and $60^{\circ} \mathrm{C}$ for 1 minute. Gene expression was calculated using the $\mathrm{CT}$ value, and fold-changes in expression were determined with the $2^{-\Delta \Delta C T}$ method.

\section{Bioinformatics analysis}

The DAVID 6.8 tool (National Institute of Allergy and Infectious Diseases, Bethesda, MD, USA) was used for Kyoto Encyclopedia of Genes and Genomes (KEGG) pathway analysis and enrichment of Gene Ontology (GO) terms for differentially expressed genes (DEGs). GO has three parts: biology processing (BP), cell components (CC), and molecules functions (MF). Moreover, STRING databases (https://string-db.org/) were used to study the interaction relationships between the proteins encoded by DEGs. Next, the protein-protein interaction (PPI) networks were visualized and edited with Cytoscape software (http://www.cytoscape.org).

\section{Lentivirus-mediated siRNA construction and infection}

An siRNA target Sox9 sequence (Sox9 siRNA, GGAGAGCGAGGAAGATAAA) was selected according to a previous study..$^{17} \mathrm{~A}$ universal sequence (negative control [NC], TTCTCCGAACGTGTCACGT) was used as a control. The siRNA sequences were cloned into a lentivirus vector GV118 carrying the green fluorescent protein gene (Genechem Co., Ltd., Shanghai, China). Lentiviral particles were prepared as previously described. ${ }^{18}$ Vectors carrying Sox 9 siRNA or NC were infected into BMSCs at a multiplicity of infection of 80. After infection for 96 hours, the cells were induced with dexamethasone and treated with BMSC Exos as previously described. Finally, the cells were used to detect the expression of Sox 9 by Western blotting and assess the effect of Sox 9 on osteogenesis by oil red $\mathrm{O}$ staining.

\section{Reverse transcription and real-time quantitative PCR (RTq-PCR)}

Total RNA was extracted using TRIzol reagent (Invitrogen). First-strand cDNA was synthesized with the $\mathrm{RT}^{2}$ First Strand Kit (Life Technologies, Carlsbad, CA, USA) following the manufacturer's protocol. RT-PCR was performed using an ABI Q6 detection system (Applied Biosystems, Foster City, CA, USA) with a Real Time SYBR master mix kit under the following conditions: $95^{\circ} \mathrm{C}, 10$ minutes; $95^{\circ} \mathrm{C}, 15$ seconds, $60^{\circ} \mathrm{C}, 1$ minute (40 cycles). The primers used for real-time quantitative PCR are shown in Table S1. The data were evaluated using the $2^{-\Delta \Delta C t}$ method, with normalization to GAPDH mRNA.

\section{Statistical analysis}

Data are shown as the mean \pm SD from three independent experiments conducted in triplicate. Statistical differences were analyzed using a paired Student's $t$-test. DEGs between two samples were identified via fold-change filtering using $P<0.05$ and a cut-off of absolute fold-change $>2$.

\section{Results Characterization of BMSC Exos}

Exos were mainly evaluated based on their size, shape, and specific proteins. TEM showed that the obtained particles from BMSCs were 100-150 nm in size and appeared homogeneous with a complete membrane structure (Figure 1A). 
A

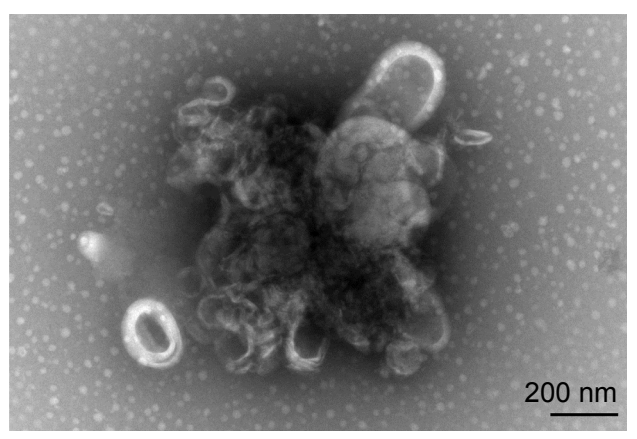

B

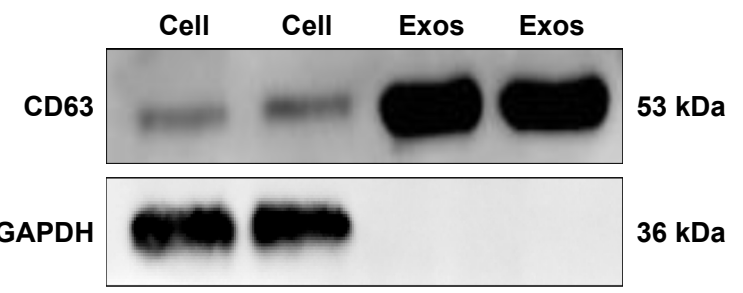

Figure I Verification of Exos from BMSCs.

Notes: (A) The morphology of Exos was analyzed by TEM (scale bar, $200 \mathrm{~nm}$ ). The size of Exos is $100-150 \mathrm{~nm}$. (B) Western blotting of CD63 was performed in BMSCs and BMSCs derived Exos. CD63 is a specific marker of Exos. $53 \mathrm{KDa}$ and $36 \mathrm{kDa}$ indicate the molecular weight of CD63 and GAPDH protein.

Abbreviations: BMSC, bone marrow mesenchymal stem cells; Exos, exosomes; TEM, transmission electron microscopy.

Western blotting revealed that the obtained particles significantly expressed CD63 protein, which is a general marker of exosomes (Figure 1B). Collectively, these results suggest that the obtained particles were BMSC Exos.

\section{Effect of BMSC Exos on osteogenesis}

Adipogenic commitment depletes the osteogenic ability of osteogenesis. To assess the effect of BMSC Exos on the osteogenic ability of BMSCs in the SFHN model, the dexamethasone-induced BMSCs were stained with oil red $\mathrm{O}$ and alizarine red. As shown in Figure 2, the lipid droplets were stained red, with more red droplets representing greater adipogenesis and fewer droplets representing less osteogenesis. The oil red $\mathrm{O}$ staining showed that the number of lipid droplets in the model group was significantly increased compared to that in the control group (Figure 2A and B). Moreover, the Exos promoted osteogenesis by depleting BMSC adipogenesis as demonstrated by a decreased oil red $\mathrm{O}$ staining. The alizarine red staining showed that osteogenic differentiation of model group was significantly decreased compared to that of control group, while BMSC Exos significantly reversed the decreased osteogenic differentiation of SFHN BMSCs.

\section{Analysis of DEGs}

To identify important genes involved in BMSC Exos effect on SFHN, gene expressions in the BMSC Exos group and model group were profiled using the Osteogenesis $\mathrm{RT}^{2}$ Profiler PCR Array. A total of 84 genes and 20 DEGs were detected. Compared to the model group, eleven and nine genes were upregulated and downregulated, respectively, in the BMSC Exos group (Figure 3). Furthermore, except for tumor necrosis factor (ligand) superfamily, member 11 (Tnfsf11), and colony-stimulating factor 2 (Csf2), whose average threshold cycles were relatively high $(>30)$, matrix metallopeptidase $9(\mathrm{Mmp} 9)$ was the most upregulated gene, followed by colony-stimulating factor 3 (Csf3), bone morphogenetic protein (Bmp2), bone morphogenetic protein receptor, type IB (Bmpr 1b), and matrix metallopeptidase 2 (Mmp2) after treatment with BMSC Exos in SFHN cells (Table S2). Moreover, the transcription factor SOX-9 (Sox9), which plays a central role in chondrocyte differentiation, was also upregulated. Additionally, the top five downregulated genes were Annexin A5 (Anxa5), bone morphogenetic protein 4 (Bmp4), bone morphogenetic protein 5 (Bmp5), cluster of differentiation 36 (Cd36), and Distal-less homeobox 5 (Dlx5).

\section{Roles of exosomal DEGs involved in SFHN}

To further investigate the roles of these DEGs, function and pathway enrichment was analyzed. In GO analysis, we found that these DEGs were involved in many processes including the BP of BMP signaling pathway, osteoblast differentiation, and immune response. Moreover, these DEGs were mainly involved in the $\mathrm{CC}$ of extracellular space, proteinaceous extracellular matrix, and membrane and MF of cytokine activity, TGF- $\beta$ receptor binding, and BMP receptor binding (Figure 4A). After mapping these genes to terms in the KEGG database, we found that the genes participated in BMP signaling pathway, TGF- $\beta$ signaling pathway, Hippo signaling pathway, hematopoietic cell lineage, pathways in cancer, and cytokine-cytokine receptor interaction (Figure 4B). Furthermore, among the DEGs, four upregulated osteogenic genes including Bmp2, Bmp6, Gdf10, and Bmprlb were involved in the BMP/TGF- $\beta$ signaling pathway.

\section{PPI network analysis of DEGs}

The PPI network for upregulated genes contained 20 nodes and 54 interactions, and the average node degree in the PPI network was 5.4. Particularly, several upregulated genes played an important role in the PPI network (Figure 4C), including BMP2, MMP9, and SOX9. Moreover, the SOX9 protein can interact with BMP2, BMP6, BMP4, and secreted phosphoprotein 1 (SPP1). Furthermore, the protein of the most upregulated gene, $M M P 9$, can interact with upregulated proteins including BMP2, BMP6, MMP2, TNFSF11, CSF2, 
A
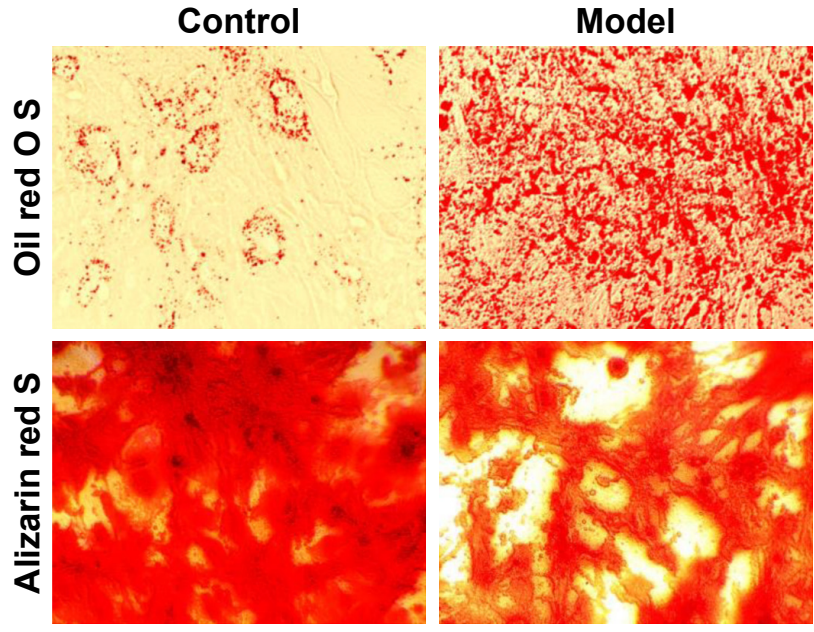

BMSC Exos

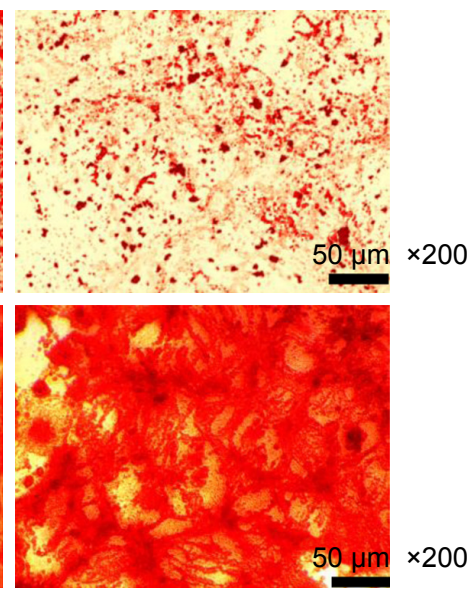

B
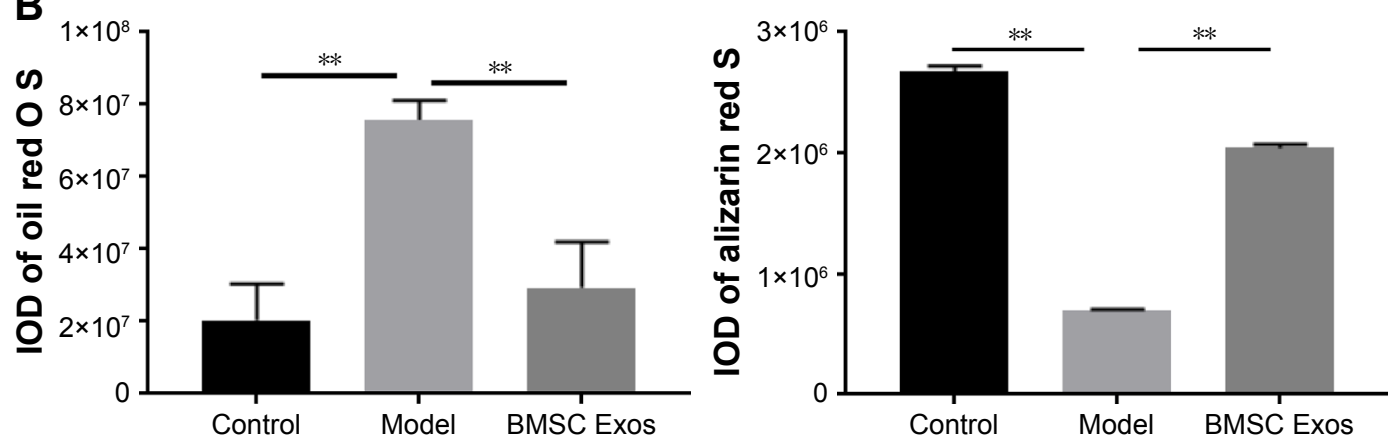

Figure 2 BMSC Exos inhibited adipogenic differentiation and enhanced osteogenic differentiation in steroid-induced BMSCs.

Notes: (A) Oil red O staining for lipid droplets (the top figures) and alizarine red staining for osteogenesis (the bottom figures) of the BMSCs from the control, model, and BMSC Exos groups. The lipid droplets were stained red, and redder droplets represented less osteogenesis. (B) Integrated OD (IOD) of lipid droplets and osteogenesis was counted using Image-pro plus. Three representative images per group were chosen for analysis. $* * P<0.0 \mathrm{I}, \mathrm{n}=3, t$-test.

Abbreviations: BMSC, bone marrow mesenchymal stem cells; Exos, exosomes.

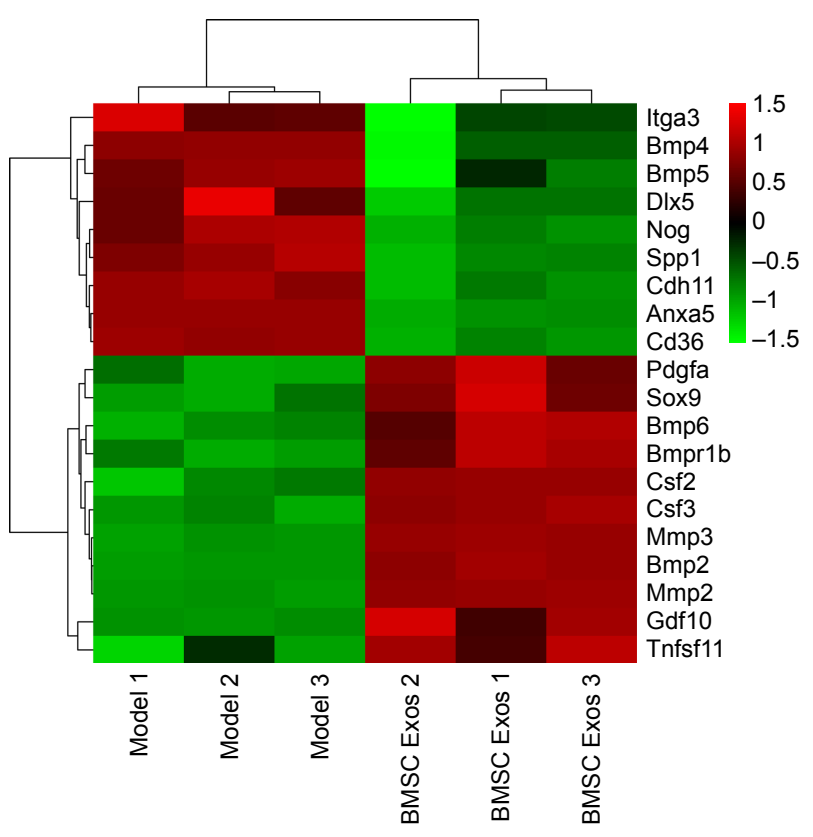

Figure 3 Hierarchical cluster analysis of differently expressed genes (DEGs).

Notes: Expression patterns of DEGs in the model and BMSC Exos groups. Each column indicates a sample and each row indicates a gene. Red strip represents high expression and green represents low expression.

Abbreviations: BMSC, bone marrow mesenchymal stem cells; Exos, exosomes. and CSF3, while downregulated proteins including BMP4, SPP1, ANXA5, and CD36 molecule (CD36).

\section{Sox9 is required for osteogenesis activation of BMSC Exos in SFHN}

As the transcription factor Sox 9 was upregulated and played a crucial role in the PPI network after BMSC Exos treatment, we examined whether $\operatorname{Sox} 9$ contributes to the osteogenic role of BMSC Exos in SFHN. The protein level of SOX9 was significantly decreased in SFHN model cells compared to that in normal cells, while BMSC Exos significantly elevated SOX9 (Figure 5, $P<0.05$ ). Using siRNA, we successfully interfered with the expression of $\operatorname{Sox} 9$ (Figure $6 \mathrm{~A}$ and B). Oil red $\mathrm{O}$ staining revealed that knockdown of $\operatorname{Sox} 9$ significantly (Figure 6C and D, $P<0.05$ ) increased the lipid droplets in the control, SFHN model, and BMSC Exo-treated cells. Furthermore, alizarine red staining displayed that Sox 9 silencing significantly abolished the osteogenic effect of BMSC Exo (Figure 6E and F, $P<0.05$ ), suggesting that the Sox9 
A

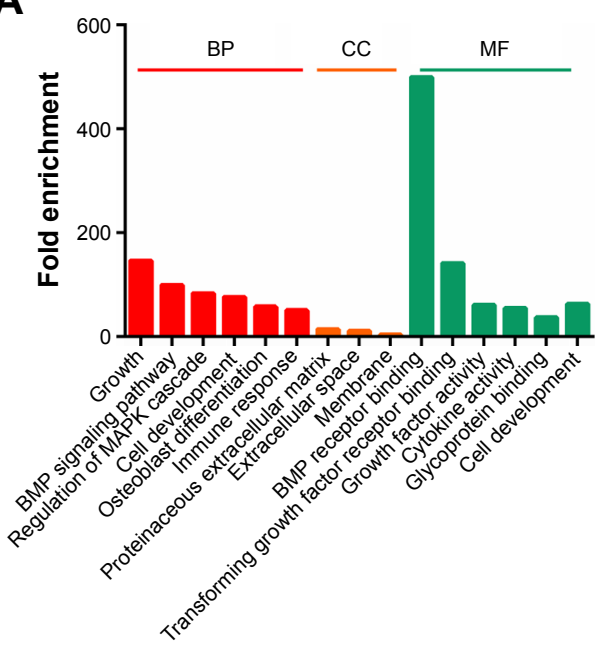

C





Figure 4 Roles of differently expressed genes (DEGs).

Notes: (A) Gene Ontology classification for DEGs induced by BMSC Exos in SFHN. The results are summarized in three categories: biological process (BP), cellular component (CC), and molecular function (MF). (B) Kyoto Encyclopedia of Genes and Genomes classification for DEGs in SFHN with incubating Exos derived from BMSCs. (C) Protein-protein interaction network constructed with DEGs. Genes with green nodes were downregulated, while red nodes were upregulated after BMSC Exos treatment in SFHN model cells.

Abbreviations: BMSC, bone marrow mesenchymal stem cells; Exos, exosomes; SFHN, steroid-induced femoral head necrosis.
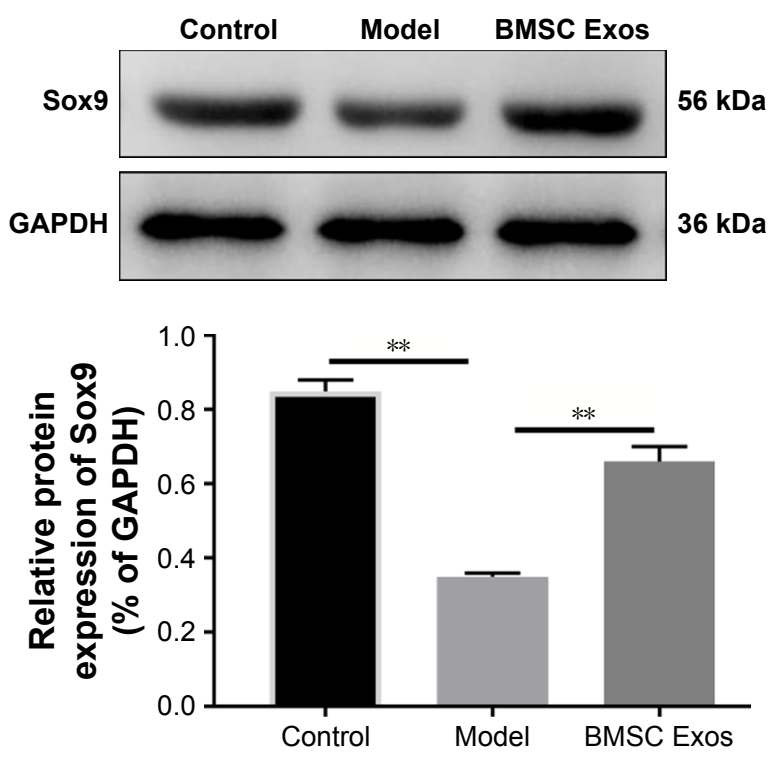

Figure 5 Protein expression of Sox 9 in BMSCs.

Notes: The protein level was measured by Western blotting and relative expression was analyzed with ImageJ software. $* * P<0.01, n=3$, $t$-test.

Abbreviations: BMSC, bone marrow mesenchymal stem cells; Exos, exosomes. promoted the osteogenesis of BMSCs and that BMSC Exos improved SFHN partly by increasing $\operatorname{Sox} 9$ expression.

\section{Discussion}

Exos play key roles in intercellular communication through the delivery of proteins, genes, and small noncoding RNAs. ${ }^{19}$ This study demonstrated that rat BMSC Exos promote the osteogenesis of SFHN. Furthermore, using the PCR array, we found that several bone formation-related genes were involved in the therapeutic action of BMSC Exos in SFHN.

Recently, several novel methods have been reported to induce directed differentiation of stem cells. For instance, Song et al demonstrated that geometry and dimensions of nanotopography significantly promote neuronal differentiation of human-induced pluripotent stem cells. ${ }^{20}$ The stiffness and nanotopographical characteristics of the extracellular matrix influence osteogenic differentiation of human 


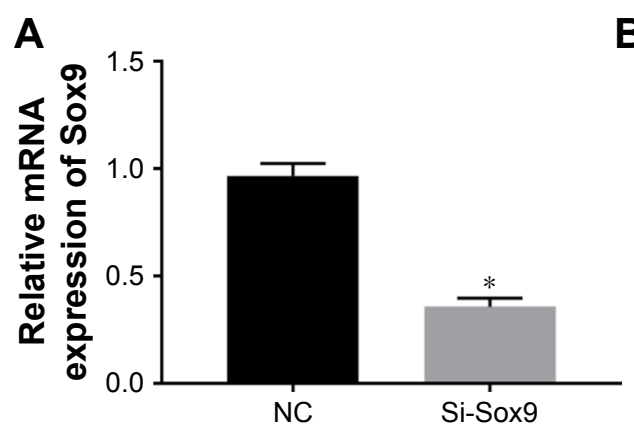

C

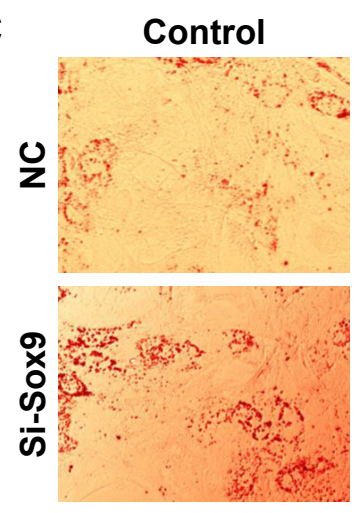

E

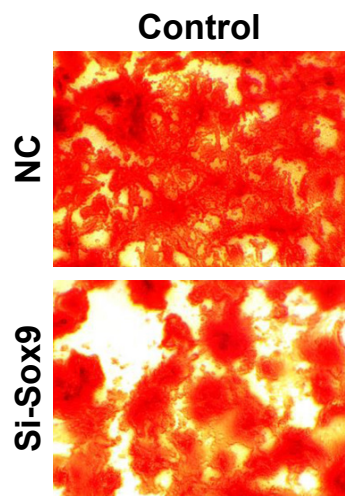

B

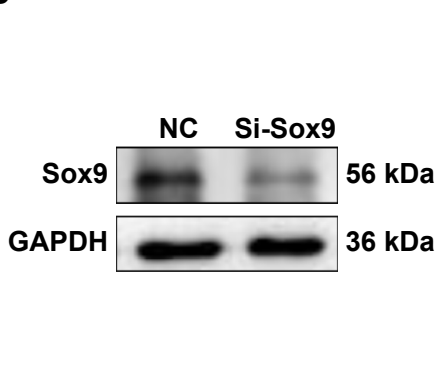

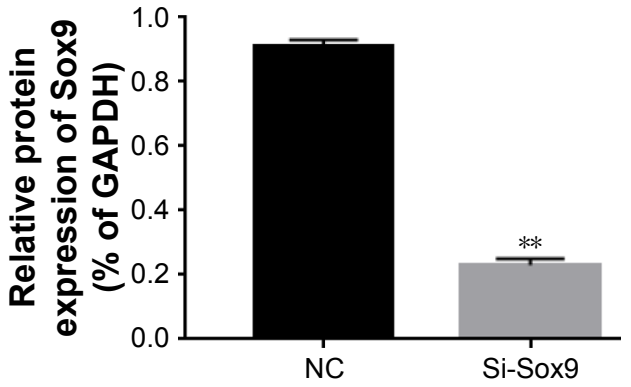

BMSC Exos


$\mathbf{F}$

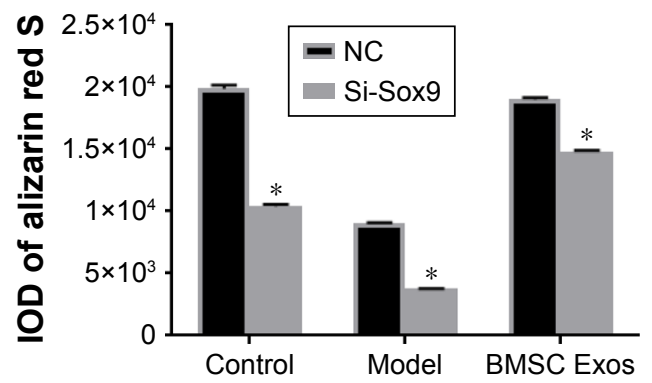

Figure 6 Effect of Sox9 on osteogenesis of BMSCs.

Notes: (A) Efficiency of Sox9 siRNA lentiviral vectors was detected by real-time quantitative PCR. (B) Efficiency of Sox 9 siRNA was detected by Western blotting. Relative expression was analyzed by ImageJ software. (C and D) Oil red O staining for lipid droplets of BMSCs. The lipid droplets were stained red, and the higher number of red droplets represents less osteogenesis. (E and F) Alizarine red staining for osteogenesis of BMSCs. Scale bar: $50 \mu \mathrm{m}$. Integrated OD (IOD) of lipid droplets and osteoblasts was counted using Image-pro plus. $* P<0.05$ compared to BMSCs transfected with control siRNA vector, $* * P<0.0$ I. This experiment was repeated three times, and statistical differences were analyzed using paired Student's t-test.

Abbreviations: BMSC, bone marrow mesenchymal stem cells; Exos, exosomes.

mesenchymal stem cells (hMSCs). ${ }^{21}$ The shape and dimensions, especially the height of nanotopography, can promote cell diffusion by focal adhesion rearrangement, thereby increasing nuclear volume, which facilitates osteogenesis and genetic medicine..$^{22}$ Moreover, the nanopore structure and the pore size influence spreading and cell shape of macrophages and subsequently modulate the inflammatory response and release the osteogenic factors, which affected the osteogenic differentiation of BMSCs. ${ }^{23}$ Compared with other nanometer therapies, Exos have more significant advantages. The composition of Exos is highly similar to that of autologous cells, so it has natural nonimmunogenicity. ${ }^{24}$ Protein composition on the surface of the Exos membrane also contributes to its excellent ability to recognize target cells. However, at present, the role of Exos in overall health and disease status is still poorly understood, so the long-term safety and therapeutic effect of Exos is still difficult to predict. Our study demonstrated that BMSC Exos promoted osteogenic differentiation of BMSCs, which might lay a foundation for the therapeutic effect of exosomes on bone disease.

An increasing number of studies have shown that Exos derived from MSCs enhance bone regeneration and reduce the severity of the disease. For instance, human embryonic MSC Exos exhibited the functions of cartilage repair and 
osteochondral regeneration in osteochondral defect rats. ${ }^{25}$ In human cartilage regeneration in vitro, BMSC Exos suppressed the adverse effects of inflammatory mediators and promoted cartilage regeneration, indicating that they can be used as a crucial medium for cartilage repair and as a new treatment for osteoarthritis. ${ }^{26}$ In a mouse model of myocardial ischemia/reperfusion injury, MSC Exos reduced infarct size and were associated with tissue injury and repair. ${ }^{27}$ Moreover, the MSC Exos affected liver protection in toxicants-induced injury, primarily by inducing proliferation and regeneration. ${ }^{10}$ Additionally, Exos from synovial-derived MSCs enhanced the proliferation and inhibited the apoptosis of MSCs. ${ }^{28}$ As observed in previous studies, our results demonstrated that BMSC Exos decreased the formation of lipid droplets by promoting osteogenesis and improving SFHN.

In this study, DEGs were mainly involved in immune response. SFHN was shown to be related to the disruption of the immune system. ${ }^{29}$ During the progression of SFHN, massive chronic inflammatory cells infiltrated the necrotic zone and tumor necrosis factor- $\alpha$-mediated alteration of M1/M2 macrophage polarization contributes to the pathogenesis of SFHN. ${ }^{30}$ A study indicated that the immune response induced via the toll-like receptor 4 signaling pathway leads to SFHN. ${ }^{31}$ This suggests that the immune response plays a pivotal role in the pathogenesis of SFHN. Additionally, upregulated osteogenesis genes including Bmp2, Bmp6, and Bmpr $1 b$ were involved in the BMP and TGF- $\beta$ signaling pathways. BMPs are a set of growth factors, originally identified as key factors in inducing the construction and development of bone and cartilage. ${ }^{32}$ Moreover, BMPs belong to the TGF- $\beta$ family, and their signal is transmitted via type I and type II serine-threonine kinase receptors and downstream effectors in the cell. ${ }^{33}$ Dysregulation of TGF- $\beta$ or BMP signaling may lead to multiple bone diseases including osteoarthritis and SFHN. ${ }^{32}$ BMPs were evaluated for treatment of FHN, and BMSCs loaded with the Bmp2 gene facilitated the repair of early-stage SFHN and its mechanical function recovery. ${ }^{34}$ BMP-VEGFloaded poly lactic-co-glycolic acid scaffolds were shown to accelerate the treatment effects of core decompression surgery and may be employed as a therapy for SFHN. ${ }^{35}$

In addition to the Bmps, Mmp 9 and $\operatorname{Sox} 9$ were also upregulated after treatment with BMSC Exos in SFHN model cells. Mmp 9 is a member of the zinc-metalloproteinases family and is related to degradation of the extracellular matrix. It has been shown that $M m p 9$ is mainly expressed via destined osteoclast precursors and osteoclasts and has a key function in bone resorption and formation. ${ }^{36}$ Consistent with our results, the mRNA level of $M m p 9$ in a mouse model of GC-induced osteonecrosis was lower compared to that in the normal group. ${ }^{37}$ A previous study showed that a single-nucleotide polymorphism of $M m p 9$ decreased the risk of FHN. ${ }^{38}$ Additionally, $\operatorname{Sox} 9$ is a transcription factor that inhibited the conversion of proliferating chondrocytes into hypertrophic chondrocytes and thus plays a central role during chondrocyte differentiation. ${ }^{39}$ Moreover, Sox 9 is a key transcription factor of Bmp2 and is involved in chondrogenesis through the BMP pathways. ${ }^{40}$ In a rabbit model, Sox9 enhanced the chondrogenesis of BMSCs, and rabbit BMSCs with Sox 9 transduction played an important role in repairing cartilage defects. ${ }^{41}$ Therefore, $M m p 9$ and $\operatorname{Sox} 9$ may play important roles in the pathogenetic process of SFHN and may affect BMSC Exo treatment of SFHN.

However, there were some limitations to the present study. These results, including the effect of BMSC Exos on osteogenesis and the genes and pathways involved in this bioprocess, require further analysis and will be evaluated in future studies.

In conclusion, our findings suggest that Exos derived from BMSCs promote osteogenesis and thus may be useful as an immunotherapeutic strategy for SFHN. A total of eleven and nine upregulated and downregulated osteogenic-related genes, respectively, were identified after treatment of SFHN samples with BMSC Exos. DEGs related to regulating the immune system processes and the BMP/TGF- $\beta$ pathway may be closely associated with the pathogenesis of SFHN. These findings provide insight into the molecular mechanisms of SFHN pathogenesis.

\section{Ethics approval and consent to participate}

This study was conducted based on our protocols that were approved by the Ethical Committee of the First Affiliated Hospital of Fujian Medical University.

\section{Availability of data and material}

The datasets used and analyzed during the current study are available from the corresponding author on reasonable request.

\section{Acknowledgments}

Thanks for all participants involved in this study. This work was sponsored by grants from the Fund of Fujian Provincial Finance Department (BPB-LJH2015-2), sponsored by the Key Clinical Specialty Discipline Construction Program of Fujian, the Youth Backbone Talent Training Program of 
Fujian Provincial Health System (2017-ZQN-48), Popularize Appropriate Technical Projects for Rural and Urban Communities of Fujian Provincial Health and Family Planning System (2017017), 2018 “13th Five-Year plan" project of Fujian Provincial Department of Education (FJJKCG18-021), Startup Fund for Scientific Research, Fujian Medical University (2017XQ1075) and Fujian Provincial Department of Health Youth Fund Project (2016-2-17).

\section{Author contributions}

PC designed the experiments. All authors contributed to data analysis, drafting and revising the article, gave final approval of the version to be published, and agree to be accountable for all aspects of the work.

\section{Disclosure}

The authors report no conflicts of interest in this work.

\section{References}

1. Chamberlain G, Fox J, Ashton B, Middleton J. Concise review: mesenchymal stem cells: their phenotype, differentiation capacity, immunological features, and potential for homing. Stem Cells. 2007; 25(11):2739-2749.

2. Fink T, Rasmussen JG, Emmersen J, et al. Adipose-derived stem cells from the brown bear (Ursus arctos) spontaneously undergo chondrogenic and osteogenic differentiation in vitro. Stem Cell Res. 2011;7(1):89-95.

3. Phinney DG, Prockop DJ. Concise review: mesenchymal stem/ multipotent stromal cells: the state of transdifferentiation and modes of tissue repair - current views. Stem Cells. 2007;25(11):2896-2902.

4. Justesen J, Stenderup K, Ebbesen EN, Mosekilde L, Steiniche T, Kassem M. Adipocyte tissue volume in bone marrow is increased with aging and in patients with osteoporosis. Biogerontology. 2001;2(3):165-171.

5. Nuttall ME, Gimble JM. Is there a therapeutic opportunity to either prevent or treat osteopenic disorders by inhibiting marrow adipogenesis? Bone. 2000;27(2):177-184.

6. Tan G, Kang PD, Pei FX. Glucocorticoids affect the metabolism of bone marrow stromal cells and lead to osteonecrosis of the femoral head: a review. Chin Med J. 2012;125(1):134-139.

7. Li J, Wang Y, Li Y, Sun J, Zhao G. The effect of combined regulation of the expression of peroxisome proliferator-activated receptor- $\gamma$ and calcitonin gene-related peptide on alcohol-induced adipogenic differentiation of bone marrow mesenchymal stem cells. Mol Cell Biochem. 2014;392(1-2):39-48.

8. Mikami T, Ichiseki T, Kaneuji A, et al. Prevention of steroid-induced osteonecrosis by intravenous administration of vitamin $\mathrm{E}$ in a rabbit model. J Orthop Sci. 2010;15(5):674-677.

9. Cook DA, Fellgett SW, Pownall ME, O'Shea PJ, Genever PG. Wntdependent osteogenic commitment of bone marrow stromal cells using a novel GSK3ß inhibitor. Stem Cell Res. 2014;12(2):415-427.

10. Tan CY, Lai RC, Wong W, Dan YY, Lim SK, Ho HK. Mesenchymal stem cell-derived exosomes promote hepatic regeneration in druginduced liver injury models. Stem Cell Res Ther. 2014;5(3):76.

11. van Niel G, Porto-Carreiro I, Simoes S, Raposo G. Exosomes: a common pathway for a specialized function. J Biochem. 2006;140(1):13-21.

12. Théry C. Exosomes: secreted vesicles and intercellular communications. F1000 Biol Rep. 2011;3:15.

13. Valadi H, Ekström K, Bossios A, Sjöstrand M, Lee JJ, Lötvall JO. Exosome-mediated transfer of mRNAs and microRNAs is a novel mechanism of genetic exchange between cells. Nat Cell Biol. 2007;9(6):654-659.
14. Skog J, Würdinger T, van Rijn $\mathrm{S}$, et al. Glioblastoma microvesicles transport RNA and proteins that promote tumour growth and provide diagnostic biomarkers. Nat Cell Biol. 2008;10(12):1470-1476.

15. Gu C, Xu Y, Zhang S, et al. miR-27a attenuates adipogenesis and promotes osteogenesis in steroid-induced rat BMSCs by targeting PPAR $\gamma$ and GREM1. Sci Rep. 2016;6:38491.

16. Huang X, Yuan T, Tschannen M, et al. Characterization of human plasma-derived exosomal RNAs by deep sequencing. BMC Genomics. 2013;14(1):319.

17. Jiang X, Huang X, Jiang T, Zheng L, Zhao J, Zhang X. The role of Sox 9 in collagen hydrogel-mediated chondrogenic differentiation of adult mesenchymal stem cells (MSCs). Biomater Sci. 2018;6(6): 1556-1568.

18. Yu LL, Chang K, Lu LS, et al. Lentivirus-mediated RNA interference targeting the $\mathrm{H} 19$ gene inhibits cell proliferation and apoptosis in human choriocarcinoma cell line JAR. BMC Cell Biol. 2013;14(1):26.

19. Potaczek DP, Michel S, Sharma V, et al. Different FCER1A polymorphisms influence IgE levels in asthmatics and non-asthmatics. Pediatr Allergy Immunol. 2013;24(5):441-449.

20. Song L, Wang K, Li Y, Yang Y. Nanotopography promoted neuronal differentiation of human induced pluripotent stem cells. Colloids SurfB Biointerfaces. 2016;148:49-58.

21. Yang Y, Wang K, Gu X, Leong KW. Biophysical regulation of cell behavior-cross talk between substrate stiffness and nanotopography. Engineering. 2017;3(1):36-54.

22. Wang K, Bruce A, Mezan R, et al. Nanotopographical modulation of cell function through nuclear deformation. ACS Appl Mater Interf. 2016;8(8):5082-5092.

23. Chen Z, Ni S, Han S, et al. Nanoporous microstructures mediate osteogenesis by modulating the osteo-immune response of macrophages. Nanoscale. 2017;9(2):706-718.

24. Ha D, Yang N, Nadithe V. Exosomes as therapeutic drug carriers and delivery vehicles across biological membranes: current perspectives and future challenges. Acta Pharm Sin B. 2016;6(4):287-296.

25. Zhang S, Chu WC, Lai RC, Lim SK, Hui JH, Toh WS. Exosomes derived from human embryonic mesenchymal stem cells promote osteochondral regeneration. Osteoarthr Cartil. 2016;24(12):2135-2140.

26. Vonk LA, van Dooremalen SFJ, Liv N, et al. Mesenchymal stromal/stem cell-derived extracellular vesicles promote human cartilage regeneration in vitro. Theranostics. 2018;8(4):906-920.

27. Lai RC, Arslan F, Lee MM, et al. Exosome secreted by MSC reduces myocardial ischemia/reperfusion injury. Stem Cell Res. 2010;4(3): 214-222.

28. Guo SC, Tao SC, Yin WJ, Qi X, Sheng JG, Zhang CQ. Exosomes from human synovial-derived mesenchymal stem cells prevent glucocorticoid-induced osteonecrosis of the femoral head in the rat. Int J Biol Sci. 2016;12(10):1262-1272.

29. Okazaki S, Nishitani Y, Nagoya S, Kaya M, Yamashita T, Matsumoto H. Femoral head osteonecrosis can be caused by disruption of the systemic immune response via the toll-like receptor 4 signalling pathway. Rheumatology. 2009;48(3):227-232.

30. Wu X, Xu W, Feng X, et al. TNF-a mediated inflammatory macrophage polarization contributes to the pathogenesis of steroid-induced osteonecrosis in mice. Int J Immunopathol Pharmacol. 2015;28(3): $351-361$.

31. Tian L, Wen Q, Dang X, You W, Fan L, Wang K. Immune response associated with Toll-like receptor 4 signaling pathway leads to steroidinduced femoral head osteonecrosis. BMC Musculoskelet Disord. 2014;15(1):1-12.

32. Papachroni KK, Karatzas DN, Papavassiliou KA, Basdra EK, Papavassiliou AG. Mechanotransduction in osteoblast regulation and bone disease. Trends Mol Med. 2009;15(5):208-216.

33. Bibbo C, Nelson J, Ehrlich D, Rougeux B. Bone morphogenetic proteins. Genes Function. 2015;1(5):287-288.

34. Tang TT, Lu B, Yue B, et al. Treatment of osteonecrosis of the femoral head with hBMP-2-gene-modified tissue-engineered bone in goats. J Bone Joint Surg Br. 2007;89(1):127-129. 
35. Zhang HX, Zhang XP, Xiao GY, et al. In vitro and in vivo evaluation of calcium phosphate composite scaffolds containing BMP-VEGF loaded PLGA microspheres for the treatment of avascular necrosis of the femoral head. Mater Sci Eng C Mater Biol Appl. 2016;60:298-307.

36. Grässel S, Beckmann J, Rath B, Vogel M, Grifka J, Tingart M. Expression profile of matrix metalloproteinase-2 and -9 and their endogenous tissue inhibitors in osteonecrotic femoral heads. Int J Mol Med. 2010; 26(1):127-133.

37. He M, Wang J, Wang G, et al. Effect of glucocorticoids on osteoclast function in a mouse model of bone necrosis. Mol Med Rep. 2016;14(2): 1054-1060.

38. du J, Liu W, Jin T, et al. A single-nucleotide polymorphism in MMP9 is associated with decreased risk of steroid-induced osteonecrosis of the femoral head. Oncotarget. 2016;7(42):68434-68441.
39. Akiyama H, Chaboissier MC, Martin JF, Schedl A, de Crombrugghe B. The transcription factor Sox 9 has essential roles in successive steps of the chondrocyte differentiation pathway and is required for expression of Sox5 and Sox6. Genes Dev. 2002;16(21):2813-2828.

40. Pan Q, Yu Y, Chen Q, et al. Sox9, a key transcription factor of bone morphogenetic protein-2-induced chondrogenesis, is activated through BMP pathway and a CCAAT box in the proximal promoter. $J$ Cell Physiol. 2008;217(1):228-241.

41. Cao L, Yang F, Liu G, et al. The promotion of cartilage defect repair using adenovirus mediated Sox 9 gene transfer of rabbit bone marrow mesenchymal stem cells. Biomaterials. 2011;32(16):3910-3920. 


\section{Supplementary materials}

Table SI Primers used for qRT-PCR in this study

\begin{tabular}{l|l|l}
\hline & Forward primer & Reverse primer \\
\hline Sox9 & CCAGCAAGAACAAGCCACAC & CTTGCCCAGAGTCTTGCTGA \\
GAPDH & GCAAGTTCAACGGCACAG & GCCAGTAGACTCCACGACA \\
\hline
\end{tabular}

Table S2 Expression of differently expressed genes in SFHN after treatment with BMSCs Exo

\begin{tabular}{|c|c|c|c|}
\hline Gene name & Folds up- or downregulation & $P$-value & Comments \\
\hline Csf2 & 225.0350106 & $2.89534 \mathrm{E}-06$ & A \\
\hline Mmp9 & 97.32764764 & 3.39033E-07 & OKAY \\
\hline Csß3 & 8.189366945 & 7.44I 27E-05 & OKAY \\
\hline Bmp2 & 6.546258615 & 1.57705E-05 & OKAY \\
\hline Tnfsfl I & 5.993083574 & 0.017099759 & A \\
\hline Bmprlb & $4.5679 \mid 586$ & 0.00411149 & OKAY \\
\hline Mmp2 & 2.945868556 & $2.74126 \mathrm{E}-07$ & OKAY \\
\hline GdfIO & 2.780458589 & 0.010445327 & A \\
\hline Bmp6 & 2.759955253 & 0.0032806 & OKAY \\
\hline Sox9 & 2.517328357 & 0.004967823 & OKAY \\
\hline Pdgfa & 2.379174467 & 0.002654345 & OKAY \\
\hline Nog & -2.033314622 & 0.000743827 & OKAY \\
\hline CdhII & -2.05404156 & 6.8722 IE-05 & OKAY \\
\hline $\operatorname{ltg} a 3$ & -2.113671544 & 0.023421016 & OKAY \\
\hline Sppl & -2.411681038 & 0.000373654 & OKAY \\
\hline Dlx5 & $-3.147 \mid 57605$ & 0.024466254 & OKAY \\
\hline $\mathrm{Cd} 36$ & $-3.394|7| 56 \mid$ & 3.71695E-06 & OKAY \\
\hline Bmp5 & -3.722572304 & 0.002588769 & OKAY \\
\hline Bmp4 & $-3.89 \mid 450343$ & 0.000205618 & OKAY \\
\hline Anxa5 & -20.4857321 & I.759/9E-08 & OKAY \\
\hline
\end{tabular}

Note: A: This gene's average threshold cycle is relatively high $(>30)$ in either the control or the test sample and is reasonably low in the other sample $(<30)$.

Abbreviations: BMSC, bone marrow mesenchymal stem cells; Exo, exosome; SFHN, steroid-induced femoral head necrosis.

Drug Design, Development and Therapy

\section{Publish your work in this journal}

Drug Design, Development and Therapy is an international, peerreviewed open-access journal that spans the spectrum of drug design and development through to clinical applications. Clinical outcomes, patient safety, and programs for the development and effective, safe, and sustained use of medicines are the features of the journal, which
Dovepress

has also been accepted for indexing on PubMed Central. The manuscript management system is completely online and includes a very quick and fair peer-review system, which is all easy to use. Visit http://www.dovepress.com/testimonials.php to read real quotes from published authors.

Submit your manuscript here: http://www.dovepress.com/drug-design-development-and-therapy-journal 